REDES- Revista hispana para el análisis de redes sociales

Vol.24,\#2, Diciembre 2013

http://revista-redes.rediris.es

\title{
El estudio del capital social de los hogares por medio de redes personales
}

Luis Portales

Universidad Cristóbal Colón, México ${ }^{1}$

\begin{abstract}
Resumen
El capital social es un concepto que si bien no cuenta con un consenso sobre su conceptualización o medición, si presenta como una de sus principales características su aspecto funcional, es decir, en todas sus aproximaciones se pone de manifiesto la potencialidad que brinda a los actores que hacen uso de él para acceder a determinados recursos, beneficios o capitales que de otra forma no podrían hacerlo. Esta característica permitió la construcción de una aproximación metodológica que, desde la visión minimalista del concepto, utilizó la técnica de redes personales como estrategia de recolección de información y un análisis de componentes principales categóricos para caracterizar el capital social de 96 hogares mexicanos en situación de pobreza. Resultado de esta aproximación fue la construcción de tres variables de capital social que tomaron en consideración la cercanía y diversidad de las relaciones sociales, y el grado de apertura de la red social de los hogares para analizar cómo este capital incide en el incremento de las condiciones de vida de los mismos, por medio del impacto que generan en su capital económico y humano.
\end{abstract}

Palabras clave: Capital social - Redes personales - Hogares - Relaciones sociales - Redes sociales.

\begin{abstract}
Social capital is a concept that although does not have a consensus on its conceptualization or measurement, presented as one of its main features its functional aspect. All approaches highlight the potential it has to provide social actors the ability to access certain resources, profits or capital that otherwise they could not. This feature was the base for the construction of a methodological approach that, taking into consideration the minimalist view of the concept use personal networks as a strategy for collecting information and categorical principal components analysis to characterize the social capital of 96 Mexican households. Result of this approach was the construction of three household's social capital variables that took into consideration the proximity and diversity of their social relations and the degree of openness of their social networks. These variables were employed to analyse how social capital increased households' conditions of life through the impact it generated in economic and human capital.
\end{abstract}

Key words: Social capital - Personal networks - Households - Social relations Social network.

1 Contacto: Luis Portales, Universidad Cristóbal Colón, México. Dirección electrónica: portales.luis@gmail.com 


\section{I ntroducción}

Las primeras conceptualizaciones del capital social datan de la década de los ochenta, sin embargo, la proliferación de estudios empíricos que lo consideran como variable explicativa de diversos fenómenos sociales como el crecimiento económico de un país, la mejora del capital humano de una comunidad o la consolidación de las instituciones en una determinada región, se presentó a partir de la década de los noventa, visión que se ha mantenido a lo largo de las primeras décadas del siglo XXI (Bartkus \& Davis, 2010; Hallinan, 2010; Millán \& Gordon, 2004; Portes \& Landolt, 2000; Robison, Siles, \& Schmid, 2003; Woolcock, 2001).

Consecuencia de esta proliferación de estudios ha sido la creación de dos visiones en la conceptualización del capital social (Ostrom \& Ahn, 2009). La primera, de corte expansionista, tiene como principal interés el estudio de los efectos y beneficios que este tipo de capital genera en la colectividad como consecuencia de la construcción de confianza, valores y normas de conducta entre sus integrantes. La segunda, de corte minimalista, se centra en el estudio de la forma en que las relaciones y redes sociales de un actor le permiten acceder a diferentes tipos de capitales o recursos.

Retomando esta última visión, el presente trabajo tiene como interés exponer una aproximación teórico-metodológica para la identificación y medición del capital social en hogares por medio de la técnica de redes personales y el Análisis de Componentes Principales Categóricos. Esta aproximación fue utilizada durante una investigación que tuvo como interés identificar el efecto y composición del capital social en hogares mexicanos en situación de pobreza ubicados en una colonia marginada del Área Metropolitana de Monterrey.

El artículo se encuentra integrado por cinco secciones. En la primera se exponen las dos visiones que existen sobre el capital social, sus principales características y las razones por las cuales la visión minimalista es la que más se adecua al estudio de este tipo de capital en los hogares. La segunda sección toma como base esta reflexión teórica para explicar el proceso metodológico que se siguió para construir las variables de capital social, desde su recolección por medio de redes personales hasta la generación de sus indicadores. En la tercera sección se presenta la forma en que se construyeron las variables de capital económico y humano que fueron introducidas en un modelo de regresión lineal que es expuesto en la cuarta sección con el interés de mostrar el impacto que el capital social genera en cada uno de ellos. 
La quinta sección presenta una serie de reflexiones entorno a los elementos de valor que presenta esta aproximación al estudio del capital social.

\section{El capital social de los hogares, una aproximación desde la visión minimalista}

La idea de capital social, entendida como la capacidad de un determinado grupo de personas para organizarse y actuar de forma colectiva que permite el acceso a beneficios concretos está presente desde la época de la Ilustración Escocesa y en la teoría sociológica clásica (Woolcock, 1998). Sin embargo, no es sino hasta la segunda década del siglo XX que surge como concepto dentro de la teoría sociológica. Posteriormente, en la década de los noventa el concepto fue impulsado por organismos internacionales que veían en él una forma de combatir la pobreza y acelerar los procesos de desarrollo de países y zonas que presentaban altos niveles de rezago socioeconómico (Bebbington, Guggenheim, Olson, \& Woolcock, 2002; Portales, 2012). Esta situación hizo que éste fuera popularizado en investigaciones empíricas de diversas disciplinas de las ciencias sociales fortaleciendo el debate teórico, metodológico y pragmático sobre dicho concepto (Millán \& Gordon, 2004; Robison et al., 2003). Este debate trajo consigo el surgimiento de dos visiones del capital social: una de tipo de expansionista y otra minimalista (Ostrom \& Ahn, 2009).

La visión expansionista tiene como interés identificar la forma en cómo las relaciones de confianza, reciprocidad, redes sociales, participación comunitaria y el seguimiento de normas de comportamiento comunes a una determinada colectividad mejoran sus condiciones de vida, ya sea por la atención a problemáticas sociales que se encuentran en el seno de la comunidad de una forma más eficiente (Coleman, 1990, 2008; Durston, 2000; Ostrom \& Ahn, 2009; Putnam, 1993; Sunkel, 2003), por su capacidad de vincularse con otros actores institucionales y posicionar sus demandas en sus agendas (Ostrom \& Ahn, 2009; Woolcock, 1998, 2001) o simplemente por la realización de acciones articuladas que están orientadas al desarrollo de la comunidad (Durston, 2002; Robison et al., 2003).

Esta visión parte del supuesto de que la colectividad tiene la capacidad de generar mecanismos de participación y de crear normas de comportamiento que operan bajo una lógica formal e informal con el interés de dar respuesta a una problemática de corte colectivo. 
La visión minimalista se centra en el análisis de la forma en que las relaciones y redes sociales generan un impacto en los actores que la conforman de una forma directa (Ostrom \& Ahn, 2009). Su análisis está centrado en el estudio de los beneficios o perjuicios que el capital social genera en actores específicos por el hecho de contar con una determinada relación social o de pertenecer a una determinada red social y no en los beneficios que genera para la colectividad. Está orientada a identificar que tipo de relaciones sociales y estructuras de determinadas redes sociales permiten a determinados actores sociales, como pueden ser los hogares, acceder a recursos y beneficios que de otra forma no podrían hacerlo.

Esta visión toma en consideración la heterogeneidad que presentan los diferentes actores que confluyen en un determinado espacio social, permitiendo conocer la forma en que el capital social incrementa el acceso a otras formas de capital y las condiciones en que lo hace. Este entendimiento permite generar una aproximación metodológica para el estudio del capital social de los hogares que se encuentran en una determinada comunidad o región.

Bajo esta visión, el capital social de un hogar es producto de las relaciones sociales que los miembros del hogar tienen por pertenecer a una determinada red social y que les da acceso a algún tipo de recurso -tangible o intangible- o capital. Es decir, está conformado por las relaciones sociales de los miembros del hogar y la red social que surge de la interacción de éstas, así como de la capacidad que estos dos componentes -relaciones y redes sociales- tienen de generar algún tipo de beneficio. Las relaciones sociales se refieren al conjunto de actores con los cuales un miembro del hogar interactúa y que permiten el acceso a ciertos beneficios o recursos. Pueden ser categorizadas de acuerdo a diversos criterios: fuerza del lazo entre dos o más personas -fuerte o débil- (Granovetter, 1973), motivaciones que generan esas relaciones (Wellman \& Wortley, 1990), rol de los actores (Woolcock, 1998) o la función que tienen en función de su antigüedad (Nahapiet \& Ghoshal, 1998). Una característica de todas ecategorizaciones es que toman en consideración la cercanía y diversidad que existe entre las diferentes relaciones sociales.

El segundo componente que conforma el capital social es el relacionado con las redes sociales, las cuales tienen que ver con el número e interacción de las relaciones sociales y la estructura de las mismas (Fuhse, 2009). La identificación de la red social permite conocer qué tipo de estructura favorece más al acceso de algún tipo de capital. En una red cerrada, donde la mayoría de los actores se conocen entre sí, 
se refuerza la homogeneidad del grupo y su identidad (Putnam, 1995; Svendsen \& Haase Sevendsen, 2009; Woolcock, 1998), mientras que en una red más abierta se permite la conexión entre actores de diferentes perfiles y la existencia de huecos estructurales (Burt, 2005).

La identificación de relaciones y redes sociales toma en consideración la heterogeneidad que presentan los diferentes hogares que confluyen en un determinado espacio social, permitiendo una mejor caracterización de su capital social y dando como resultado la posibilidad de establecer relaciones de causalidad o correlación con otras variables según los intereses que persiga una determinada investigación.

Un aspecto importante en el entendimiento del capital social y su aspecto funcional, identificado por su capacidad por ser transformado bajo determinadas circunstancias en otras formas de capital (Bourdieu, 1985). Es decir, el capital social tiene la capacidad de brindar acceso o de impactar en otras formas de capital por medio de su movilización o creación. Como consecuencia de esta naturaleza relacional y funcional este capital ha sido considerado como el principal activo con el que cuentan las poblaciones en situación de pobreza o rezago social para salir de dicha condición, pues a través de él se accede a recursos tangibles e intangibles que de otra forma sería poco probable obtener (Rojas, 2010; Social Capital Initiative, 1998; Stiglitz, Sen, \& Fitoussi, 2008).

Tomando como referencia este entendido, se puede establecer que el capital social de los hogares genera un impacto en el capital económico de los mismos en la medida en que las relaciones sociales o la pertenencia a una determinada red social permiten a los miembros del hogar acceder a recursos o bienes materiales (Tabla 1 ). Del mismo modo, el capital humano puede ser evaluado en función de las habilidades y conocimientos que un determinado miembro del hogar obtuvo por pertenecer a una red social o por contar con cierto tipo de relaciones sociales (Tabla 1). 


\begin{tabular}{|c|c|c|c|}
\hline Visión & Capital Social & Capital Económico & Capital Humano \\
\hline Minimalista & $\begin{array}{l}\text { Existencia de relaciones de } \\
\text { confianza y solidaridad. } \\
\text { Apertura o cierre de la red } \\
\text { social }\end{array}$ & $\begin{array}{l}\text { Acceso de los miembros a } \\
\text { recursos materiales por su } \\
\text { pertenencia a alguna red } \\
\text { social. }\end{array}$ & $\begin{array}{l}\text { Desarrollo de capacidades, } \\
\text { habilidades } \\
\text { conocimientos entre los } \\
\text { miembros del grupo. }\end{array}$ \\
\hline
\end{tabular}

Tabla 1. Evaluaciones del capital social por el impacto generado según la visión minimalista.

Es así que el capital social, desde una visión minimalista, puede ser evaluado tomando como base la cercanía y tipo de relaciones sociales que presentan los actores que conforman una determinada red social, así como del grado de cierre o apertura que presentan las redes sociales que son estructuradas por dichas relaciones sociales. Evaluación que permite la creación de tipologías o indicadores de capital social que favorecen a su caracterización.

A la par de la identificación de los tipos de capital social que existen, es necesario identificar el impacto que cada uno de ellos genera en el acceso a otras formas de capital, tal como puede ser el económico y el humano. De esta forma se puede comprender los efectos que cada tipo de capital social genera en los actores que hacen uso de él. Para lograr este interés es necesario seguir una metodología de recolección de información que permita visualizar estos aspectos tal como se menciona a continuación.

\section{Construcción de variables del capital social de los hogares}

El trabajo de campo de esta investigación se llevó a cabo de febrero del 2010 a junio del 2011 en la colonia Fernando Amilpa, ubicada en el Área Metropolitana de Monterrey y que se caracteriza por las condiciones de pobreza y los altos índices de rezago social en que se encuentran las mayor parte de sus hogares: alrededor del $70 \%$ se encuentran en algún tipo de pobreza (CONEVAL, 2012), el 58\% tienen índices de rezago social considerados "muy altos" y el $42 \%$ presenta índices "altos" (Martínez, Treviño, y Gómez, 2009).

La colonia contaba con planteles de educación pre-escolar, primaria y secundaria, un centro de salud y un Centro Comunitario de Desarrollo Social (CCDS), el cual es administrado por el gobierno estatal y funge como eje de la política social, 
concentrando su labor en la realización de talleres de capacitación para niños, jóvenes y adultos. Asimismo, la mayor parte de los hogares -alrededor del $80 \%$ contaban con cobertura de servicios básicos (luz, drenaje, pavimentación en su calle y agua).

El trabajo de campo se realizó en dos etapas. La primera fue observación participante -en donde el autor trabajó como facilitador voluntario en los Centros de Desarrollo Social de la colonia- durante los meses de marzo de 2010 a noviembre de 2010. Posteriormente se llevaron a cabo entrevistas estructuradas a una muestra aleatoria de 96 hogares, de un universo de 1,300 dando una confianza del 95\% y un error del 0.1. En dichas entrevistas se abordaron aspectos pertenecientes al capital económico, humano y social de los hogares a través de la reconstrucción de la historia del hogar. Para la conformación del capital económico se consideraron las dimensiones de ingresos, aspectos laborales y calidad de la vivienda (Rodríguez y Kinto, 2010), mientras que para el caso del capital humano se consideró la educación y el acceso a la salud (Sen, 1981).

A nivel del capital social se retomó la óptica minimalista para definirlo como la suma de las relaciones sociales que presenta un determinado actor y las redes sociales que producen la interacción de estas relaciones. Para que este entendimiento pudiera ser aplicado a nivel de los hogares fue necesario identificar las relaciones sociales y las redes sociales que los diferentes miembros del hogar habían utilizado para tener algún tipo de beneficio. En el caso de esta investigación se tomó como beneficio el acceder a los diferentes componentes que conforman el capital económico y humano de los hogares.

En aras de logar esta identificación de capital social se utilizó la técnica de redes personales, la cual permite conocer los actores con los cuales se relaciona un actor en una determinada situación. En el caso de este trabajo se tomó como actor central o "ego" al hogar y como "alteri" a las relaciones sociales que utilizan para acceder a otras formas de capital.

Para lograr la aplicabilidad de la técnica de redes personales a una unidad de análisis como es el hogar fue necesario definir como unidad de observación a aquellas personas que son miembros del hogar y que aportan al gasto del mismo, siendo la suma de las relaciones sociales mencionados por ellos y la red social producto de la interacción que tienen entre sí los elementos que conforman el capital social del hogar. En este punto es importante recordar que estas relaciones y redes deben de 
permitir el acceso a algún tipo capital para que sea considerado como una forma de capital social.

Tradicionalmente, en la técnica de redes personales no se conoce con anterioridad el nombre de los alteri o relaciones sociales a los que el ego, en este caso miembros del hogar, hará referencia (McCarty \& Govindaramanujam, 2005). Por esta razón, el generador de nombres juega un rol importante en la adquisición de la información (McCallister \& Fischer, 1978). Debido a que la investigación buscaba generar nombres o alteri según el tipo de capital al que brindaba acceso se utilizó un generador de nombres múltiple, el cual permitió que conforme se preguntaba sobre el proceso que había seguido el hogar en la conformación de su capital económico o humano se fueran identificando los alteri que participaban en el acceso a cada uno de los componentes que los conformaban.

Además, se optó por no contar con un número de alteri previamente definidos, sino que más bien fueran libres en función de la propia historia del hogar y la conformación de sus capitales. Esta estrategia obedece al entendido que se tiene sobre el capital social de los hogares en situación de pobreza, en donde se presupone que un mayor número de relaciones equivale a una mayor cantidad de este tipo de capital y por ende a una mayor capacidad de movilizar otros tipos de recursos (Collier, 1998; Durston, 2002; Grootaert, Narayan, Jones, \& Woolcock, 2003; Moser, 1998; Narayan, 1997, 1999; Social Capital Initiative, 1998; Woolcock, 1998)

El interpretador de nombres estuvo conformado por tres variables ordinales: Tipo de relación, frecuencia de la interacción y espacio geográfico de la interacción. Las categorías de cada una de las variables están relacionadas con la cercanía o lejanía que el alter tiene con el ego (Tabla 2). El valor asignado a cada una de las categorías fue definido en función de la cercanía que cada uno de los miembros de los hogares manifestaron y con base en la identificación del contexto bajo el cual fueron mencionados. Para ello se le preguntó a todos los miembros de los hogares entrevistados en que lugar situarían cada una de las categorías que conformaban el interpretador de nombres con respecto a las demás, siendo la de menor valor la considerada más cercana al hogar y la de mayor valor la más alejada. 


\begin{tabular}{|c|c|c|c|}
\hline Atributo & $\begin{array}{c}\text { Valor } \\
\text { asignado }\end{array}$ & Tipo & Cercanía al hogar \\
\hline \multirow{6}{*}{ 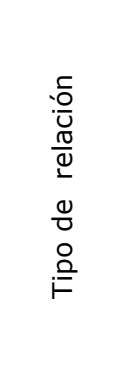 } & 1 & Familiar & \multirow{6}{*}{ Baja Cercanía } \\
\hline & 2 & Amigo & \\
\hline & 3 & Vecino & \\
\hline & 4 & Líder & \\
\hline & 5 & Privado / Laboral & \\
\hline & 5 & Gobierno / Institucional & \\
\hline \multirow{5}{*}{ 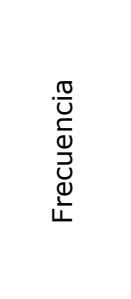 } & 1 & Diario & \multirow{5}{*}{ Baja Cercanía } \\
\hline & 2 & Semanal & \\
\hline & 3 & Mensual & \\
\hline & 4 & Ocasional & \\
\hline & 5 & Necesidad / Puntual & \\
\hline \multirow{8}{*}{ 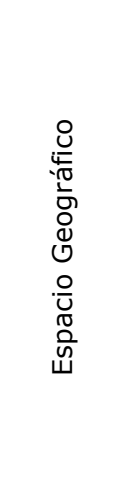 } & 1 & Casa & \multirow{8}{*}{ Alta cercanía } \\
\hline & 2 & Calle & \\
\hline & 3 & Colonia & \\
\hline & 4 & Escuela & \\
\hline & 4 & Centro Salud & \\
\hline & 5 & CCDS & \\
\hline & 6 & Gobierno & \\
\hline & 6 & Trabajo & \\
\hline
\end{tabular}

Tabla 2. Ponderado de los atributos de las relaciones sociales

Resultado de este ejercicio fue la existencia de categorías que fueron consideradas por los entrevistados a la misma distancia, es decir, que se encontraban en el mismo valor. De ahí que en el caso de la variable "tipo de relación" la categoría privado y gobierno fueran considerados en el mismo nivel de lejanía. Situación similar se presentó en la variable de "espacio geográfico" donde las categorías de escuela y centro de salud ocuparon el cuarto lugar, mientras que las variables de gobierno y trabajo ocuparon el sexto lugar (Tabla 2). En este punto es importante mencionar que si un mismo alter era mencionado por varios miembros del hogar estos no recibían una ponderación especial, sino que se tomaba en consideración la categorización hecha por el dueño de la vivienda, también denominado como jefe o jefa de familia o proveedor principal. 
Al integrar el generador e interpretador de nombres en la entrevista se pudieron identificar no solo los alteri del hogar, sino también el contexto bajo el cual eran mencionados y la forma en que permitían el acceso al capital económico y humano. De este modo se obtuvo una mayor cantidad de información etnográfica vinculada con la utilización del capital social como estrategia de vida de los hogares.

Una vez identificados los alteri con que se relacionan los diferentes miembros del hogar se partió de la idea de estructura social cognitiva para identificar la red social del hogar (Krackhardt, 1987). Para lograrlo se pidió a los miembros del hogar que mencionaran cuáles alteri tenían algún tipo de relación entre sí, dando como resultado la red social del hogar. Para la sistematización de la información de las relaciones sociales se utilizó el software Egonet para su captura y posteriormente se pasó a formato Ucinet para su análisis.

Con la identificación de los alteri que permitieron a algunos de los miembros del hogar el acceso a algún tipo de capital -llámese económico o humano- y el establecimiento de las relaciones que guardaban entre sí y que daban como resultado la red social de los hogares, se tuvieron los elementos suficientes para construir las variables que caracterizaron al capital social de los hogares y que son descritas a continuación.

\section{Las variables del capital social}

Se construyeron tres variables relacionadas con el capital social mismas que conforman las variables independientes del modelo estadístico utilizado. Las primeras dos variables se construyeron con base al grado de cercanía o lejanía de los alteri o relaciones sociales del hogar. La construcción de estas dos variables tuvo como base la generación de un indicador que dio muestra de la cercanía o lejanía de las relaciones sociales de los hogares en función del tipo de capital al que dan acceso.

Este indicador fue denominado Índice de Proximidad de Relaciones Sociales (IPRS) y es un aproximado a la idea de Granovetter (1973) sobre lazos fuertes y débiles, así como de Putnam (1995) y Woolcock (2001) sobre los tipos de capital social: cierre, puente y enlace. Se diferencia de estos indicadores en el hecho de que no se presenta de forma dicotómica, como en el caso de Granovetter y Putnam, o categórica, como en el caso de Woolcock, sino que es un continuo en donde los lazos débiles o de enlace se encuentran en la parte alta del indicador, es decir, que tienen 
un IPRS más elevado; mientras que los lazos fuertes o relación de cierre en la parte baja, es decir, tienen un IPRS más bajo. El desarrollo de un indicador continuo y no categórico, reduce la incertidumbre sobre dónde colocar una relación que por sus características sea difícil de ubicar como un lazo fuerte o débil, o bien de cierre o puente.

En la construcción del IPRS se utilizó el Análisis de Componentes Principales Categóricos (CATPCA, por sus siglas en inglés). Este procedimiento de cuantificación o escalamiento óptimo se utiliza cuando las variables son de naturaleza categórica, por lo que se deben hacer adaptaciones y así proceder a aplicar un modelo factorial. La solución de un análisis de componentes principales categórico maximiza las correlaciones de las puntuaciones de objetos con cada una de las variables cuantificadas para el número de componentes (dimensiones) especificado. El CATPCA es una alternativa al cálculo de las correlaciones entre las escalas no numéricas categóricas u ordinales- y su análisis mediante un enfoque de análisis de factores o de componentes principales estándar (Gadermann, Guhn, \& Zumbo, 2012).

EI CATPCA permitió integrar tres variables categóricas en un solo indicador, mismo que denotaba el grado de cercanía o lejanía que tenían los alteri con respecto al hogar. Las categorías de cada una de las variables están relacionadas con la cercanía o lejanía que el alter tiene con el hogar (ver Tabla 2). Por ejemplo, un alter cercano al hogar con un IPRS más bajo, es aquel que tiene una relación familiar, con una frecuencia diaria y tiene como espacio de interacción la casa o vivienda. Situación opuesta es la que presenta una relación que pertenece al gobierno, con una frecuencia de interacción catalogada como necesidad y en un espacio geográfico de interacción del gobierno -como pueden ser las oficinas públicas-, la cual será catalogada como lejana al hogar y tendrá un IPRS más elevado.

En la construcción del IPRS se tomaron en consideración todos los alteri que fueron mencionados por los miembros de los 96 hogares entrevistados dando un total de 2,095. Los valores del IPRS son aquellos emanados del primer componente principal del análisis estadístico, de tal forma que cada uno de los alteri mencionados tiene un valor según la cercanía o lejanía que presentan con el hogar. El resultado del CATPCA para la integración de las tres variables del IPRS tuvo un alfa de Cronbach de 0.86, aspecto que da muestra de la consistencia interna del indicador (Gadermann et al., 2012; Oviedo \& Campo Arias, 2005). Es decir, las variables incluidas en el indicador están lo suficiente relacionadas entre sí como para medir el 
mismo constructo, en este caso la cercanía o lejanía de las relaciones sociales. El indicador explica el $78 \%$ de la varianza observada en cada una de las variables, las cuales tienen una correlación significativa entre ellas dando muestra del hecho que evalúan cosas similares (Tabla 3).

\begin{tabular}{|c|c|c|c|c|c|}
\hline \multirow{2}{*}{ Variable } & \multicolumn{3}{|c|}{$\begin{array}{c}\text { Correlaciones de las Variables } \\
\text { transformadas }\end{array}$} & \multicolumn{2}{|c|}{$\begin{array}{l}\text { Indicadores de } \\
\text { confiabilidad }\end{array}$} \\
\hline & Espacio & Frecuencia & Relación & $\begin{array}{l}\text { Alfa de } \\
\text { Cronbach }\end{array}$ & $\begin{array}{l}\% \text { de la } \\
\text { varianza }\end{array}$ \\
\hline Espacio & 1 & & & \multirow{3}{*}{0.86} & \multirow{3}{*}{78.081} \\
\hline Frecuencia & $.198^{* *}$ & 1 & & & \\
\hline Relación & $.528^{* *}$ & $.413^{* *}$ & 1 & & \\
\hline $\mathrm{N}=2,095$ & & & & & \\
\hline
\end{tabular}

Tabla 3. Resumen del modelo para la construcción del IPRS

Ahora bien, dado que el IPRS es un indicador que está referenciado a los alteri y no a los hogares que es la unidad de análisis deseada, se hizo necesaria la construcción de dos variables que sirvieran para hacer referencia a las relaciones sociales de los hogares.

La primera de ellas se concentró en la cercanía o lejanía que presentan la mayor parte de las relaciones de los hogares, denominado Indicador de Cercanía en las Relaciones Sociales del Hogar ( $\mathrm{CRH})$. En su construcción se utilizó el promedio del IPRS de todos los alteri que habían sido mencionados por los miembros de un mismo hogar. Los hogares que tienen un $\mathrm{CRH}$ menor tienen un mayor número de relaciones con familiares, con una frecuencia diaria y al interior de la vivienda. Mientras que los hogares con un $\mathrm{CRH}$ alto presentan un mayor número relaciones sociales con líderes o gente de gobierno, de una forma ocasional o por necesidad, y que se llevan a cabo afuera de la colonia.

La segunda variable que se construyó tomó en consideración la diversidad en las relaciones sociales de los hogares y fue denominada Indicador de Diversidad de las Relaciones Sociales del Hogar (DRH). En su formulación se utilizó la desviación estándar del IPRS de los alteri que fueron mencionados por los miembros de cada uno de los hogares. Un hogar con un DRH mayor será aquel que tenga mayor diversidad en el tipo de relaciones sociales, mientras que uno con un DRH menor será el que tenga relaciones sociales más semejantes entre sí. 
De este modo se construyeron dos variables relacionadas con el componente de relaciones sociales del capital social para cada uno de los hogares, mismas que fueron introducidas en el modelo de análisis que se presentará posteriormente.

La tercera variable de capital social tomó en consideración el grado de cohesión que presenta la red social del hogar. Para ello se utilizó la medida de densidad de la red, la cual es definida como la relación entre el número de lazos que existen entre los miembros de la red y el número lazos posibles de la misma (Hanneman \& Riddle, 2005). Sus valores oscilan entre cero y uno. Un hogar con una densidad alta o tendiente a uno presenta una red en donde la mayor parte de sus miembros se conocen y tienen mayor interacción entre sí, mientras que una red con baja densidad o tendiente a cero es aquella donde los alteri difícilmente se conocen entre sí.

De esta forma el capital social de los hogares fue caracterizado por tres diferentes variables: Cercanía en las Relaciones Sociales del Hogar (CRH), Diversidad de las Relaciones Sociales del Hogar (DRH) y la Densidad de la Red Social del Hogar (D). Estas tres variables conforman, además del capital social de los hogares, las variables dependientes del modelo de regresión lineal que se utilizó para visualizar el efecto del capital social de los hogares en su capital económico y humano.

Con la intención de validar que estas variables no presentaran rasgos de multicolinealidad que pudieran afectar la regresión lineal, se calcularon los estadísticos de tolerancia y Factor de Inflación de la Varianza (FIV). En el caso de la tolerancia se encontró que los valores fueron cercanos a uno, es decir, estuvieron cercanos a su valor máximo. Situación opuesta se presentó en los Factores de Inflación de la Varianza, donde los valores fueron cercanos a uno que es su valor mínimo (Tabla 4). Los valores en ambos indicadores dan muestra de una ausencia de colinealidad entre las variables del capital social, por lo cual pueden ser utilizadas sin problema en el modelo de regresión lineal que se define en la siguiente sección.

\begin{tabular}{|l|c|c|}
\hline \multirow{2}{*}{ Variable de capital social } & \multicolumn{2}{c|}{ Estadísticos de colinealidad } \\
\cline { 2 - 3 } & Tolerancia & FIV \\
\hline Densidad (D) & 0.986 & 1.014 \\
\hline $\begin{array}{l}\text { Indicador Cercanía de las relaciones } \\
\text { sociales (CRH) }\end{array}$ & 0.714 & 1.401 \\
\hline $\begin{array}{l}\text { Diversidad en las relaciones sociales } \\
\text { (DRH) }\end{array}$ & 0.718 & 1.392 \\
\hline
\end{tabular}

Tabla 4. Pruebas de colinealidad entre las variables de capital social 


\section{El capital económico y humano de los hogares}

El primer índice que se realizó fue el del capital económico. Incluyó siete diferentes variables que están vinculadas con tres dimensiones: ingresos, aspectos laborales y características de la vivienda (Tabla 5).

En relación a los ingresos del hogar se utilizaron dos variables, la primera de ellas fueron los ingresos que recibe el hogar de forma constante y la segunda son los ingresos que recibe de manera informal o esporádica por la realización de alguna actividad productiva adicional a la principal fuente de ingresos del hogar. La variable de aspectos laborales contempla el promedio de prestaciones laborales que reciben los integrantes del hogar que aportan al gasto: aguinaldo, vacaciones, reparto de utilidades, seguridad social y crédito para la vivienda ${ }^{2}$. Las características de la vivienda contemplan tres variables relacionadas con el material con que está construida (paredes, techo y piso) -valores del uno al cuatro según el tipo de material-, y una cuarta que contempla el promedio de servicios de infraestructura básica con que cuenta la vivienda: agua corriente, sanitario, drenaje, luz eléctrica y combustible ${ }^{3}$.

\footnotetext{
${ }^{2}$ Cada prestación otorgada es sumada al total de prestaciones del hogar, mismas que son divididas entre el número de personas que aportan al hogar, siendo el mínimo cero y el máximo cinco.

${ }^{3}$ Cada servicio con que cuenta el hogar es sumado al nivel de infraestructura básica del hogar. El número máximo es seis y el mínimo cero.
} 


\begin{tabular}{|c|c|c|}
\hline Dimensión & Variable & Valores de la variables \\
\hline \multirow{2}{*}{ Ingresos } & Ingresos per cápita del hogar & Ingresos monetarios por semana \\
\hline & Ingresos per cápita adicionales del hogar & Ingresos monetarios por semana \\
\hline Aspectos laborales & Prestaciones sociales & $\begin{array}{l}0=\text { ninguna prestación } \\
1=\text { una prestación } \\
2=\text { dos prestaciones } \\
3=\text { tres prestaciones } \\
4=\text { cuatro prestaciones }\end{array}$ \\
\hline \multirow{4}{*}{ Vivienda } & Techo & $\begin{array}{l}\text { 1. Material de desecho } \\
\text { 2. Lámina } \\
\text { 3. Madera o terrado con vigas } \\
\text { 4. Losa de concreto }\end{array}$ \\
\hline & Piso & $\begin{array}{l}\text { 1. Tierra } \\
\text { 2. Cemento o firme } \\
\text { 3. Mosaico o vinil }\end{array}$ \\
\hline & Paredes & $\begin{array}{l}\text { 1. Material de desecho } \\
\text { 2. Lámina } \\
\text { 3. Madera } \\
\text { 4. Adobe }\end{array}$ \\
\hline & Infraestructura básica & $\begin{array}{l}0=\text { ninguna servicio } \\
1=\text { un servicio } \\
2=\text { dos servicios } \\
3=\text { tres servicios } \\
4=\text { cuatro servicios } \\
5=\text { cinco servicios } \\
6=\text { seis servicios }\end{array}$ \\
\hline
\end{tabular}

Tabla 5. Variables incluidas dentro del indicador de capital económico por dimensiones.

Al igual que en el caso del IPRS se utilizó el CATPCA para generar un indicador de capital social, esto por su facilidad de maximizar las correlaciones de las puntuaciones de objetos con cada una de las variables cuantificadas para el número de dimensiones especificado. La mayor parte de las variables muestran correlaciones con significancias del .01 y 0.05 entre ellas. El resultado de la aplicación del modelo arrojó un alfa de Cronbach de 0.711 , aspecto que habla de la consistencia interna que tiene el indicador y explica el $36.6 \%$ de la varianza de las variables incluidas en el modelo (Tabla 6). 


\begin{tabular}{|c|c|c|c|c|c|c|c|c|c|c|}
\hline \multirow{2}{*}{\multicolumn{2}{|c|}{ Variable }} & \multicolumn{7}{|c|}{ Correlaciones de las Variables transformadas } & \multicolumn{2}{|c|}{$\begin{array}{l}\text { Indicadores de } \\
\text { confiabilidad }\end{array}$} \\
\hline & & 1 & 2 & 3 & 4 & 5 & 6 & 7 & $\begin{array}{l}\text { Alfa de } \\
\text { Cronbach }\end{array}$ & $\begin{array}{l}\% \text { de la } \\
\text { varianza }\end{array}$ \\
\hline 1 & Aspectos Laborales & 1 & & & & & & & & \\
\hline 2 & $\begin{array}{l}\text { Ingreso per cápita por } \\
\text { semana }\end{array}$ & -.038 & 1 & & & & & & & \\
\hline 3 & $\begin{array}{l}\text { Ingresos per cápita por } \\
\text { semana extras }\end{array}$ & -.039 & $.278^{* *}$ & 1 & & & & & 711 & 36610 \\
\hline 4 & Paredes & .108 & .009 & .011 & 1 & & & & .111 & 30.010 \\
\hline 5 & Techo & $.189^{* *}$ & .116 & .094 & $.377^{* *}$ & 1 & & & & \\
\hline 6 & Piso & -.117 & .095 & $.155^{*}$ & $.205^{* *}$ & $.292^{* *}$ & 1 & & & \\
\hline 7 & Infraestructura vivienda & $.242^{* *}$ & $.294^{* *}$ & $.161^{*}$ & $.302^{* *}$ & $.497^{* *}$ & $.344^{* *}$ & 1 & & \\
\hline \multicolumn{11}{|c|}{$N=96$} \\
\hline \multicolumn{11}{|c|}{ **. La correlación es significativa al nivel 0,01 (bilateral). } \\
\hline
\end{tabular}

Tabla 6. Resumen del modelo para la construcción del indicador de capital económico

En la construcción del indicador de capital humano se consideraron ocho variables diferentes de las cuales cuatro se encuentran dentro de la dimensión educativa y cuatro en la dimensión de salud (Tabla 7). Si bien es cierto que tradicionalmente la variable educativa ha sido vinculada con el capital humano, también es cierto que en aproximaciones actuales al fenómeno de la pobreza y la calidad de vida, la salud ha sido incluida como una elemento clave en la conformación de este capital tal como lo confirman las aproximaciones de Sen (1981), Stiglitz, Sen \& Fitoussi (2008), Esposito \& Chiappero-Martinetti (2010), entre otros.

En la dimensión educativa, las variables que se utilizaron pueden ser dividas en dos, la primera se refiere a la educación formal y la segunda está bien vinculada con la educación no escolarizada. En el aspecto formal se encuentran las variables que contemplan el promedio de escolaridad de las personas que aportan al gasto y/o no estudian y el máximo grado alcanzado por el jefe o jefa de familia. Desde un punto de vista no escolarizado, el indicador contempla las variables de cursos adicionales que han tomado los miembros del hogar fuera del sistema educativo formal y el número de mayores de edad que tomaron cursos de capacitación o de formación después de haber finalizado sus estudios.

En la dimensión de acceso a la salud se tomaron en consideración cuatro diferentes variables orientadas a identificar las posibles condiciones de vulnerabilidad en que se encuentran los hogares. La primera variable es "la primera opción a la que acuden 
cuando tienen algún problema de salud". Contempla como un menor grado de vulnerabilidad el acudir a alguna instalación de gobierno para atenderse ya que no genera ningún tipo de gasto para el hogar, situación que es relevante por la situación de pobreza que presentan y que ha sido abordada en varios estudios vinculados con esta problemática en términos de política pública ${ }^{4}$.La segunda variable identifica la razón por la cual los miembros del hogar deciden acudir a este servicio de salud. Esta variable retoma la idea de que la situación de vulnerabilidad que presentan los hogares en situación de pobreza hace que éstos no sean capaces de decidir el tipo de servicio de salud al que acceden, disminuyendo así su calidad de vida y la posibilidad de mejorar las condiciones que presentan. La tercera variable es la relación que presenta el hogar entre el número de afiliados a algún sistema de salud y los que no. La última y cuarta variable está vinculada con el tiempo de espera para ser atendidos cuando llegan a atenderse a alguna institución de salud (Tabla 7).

\begin{tabular}{|c|c|c|}
\hline Dimensión & Variable & Valores de la variables \\
\hline \multirow{4}{*}{ Educativa } & $\begin{array}{l}\text { Escolaridad de los } \\
\text { mayores que aportan al } \\
\text { gasto }\end{array}$ & $\begin{array}{l}\text { Promedio de escolaridad alcanzado por los mayores de } \\
\text { edad que aportan al gasto }\end{array}$ \\
\hline & $\begin{array}{lll}\text { Máximo } \\
\text { estudios }\end{array}$ & $\begin{array}{l}\text { Máximo grado de estudios alcanzado por el jefe o jefa de } \\
\text { familia }\end{array}$ \\
\hline & Cursos adicionales & $\begin{array}{l}0 \text { cursos }=\text { cero } \\
1-3 \text { cursos = uno } \\
4-6=\text { dos } \\
<6=\text { tres. }\end{array}$ \\
\hline & $\begin{array}{l}\text { Mayores que tomaron } \\
\text { cursos adicionales }\end{array}$ & $\begin{array}{l}\text { Relación de personas mayores de edad que tomaron } \\
\text { cursos adicionales al sistema educativo formal entre los } \\
\text { que no. }\end{array}$ \\
\hline \multirow{4}{*}{ Salud } & $\begin{array}{l}\text { Primera opción para } \\
\text { atenderse }\end{array}$ & $\begin{array}{l}\text { 1. IMSS o ISSSTE } \\
\text { 2. Centro de Salud } \\
\text { 3. Hospital o Instituto de la Secretaría de Salud) } \\
\text { 4. Consultorio u hospital privado }\end{array}$ \\
\hline & $\begin{array}{l}\text { Razón por que acude a } \\
\text { ese servicio de salud }\end{array}$ & $\begin{array}{l}\text { 1. Es bueno } \\
\text { 2. Es bueno y es el único al cual acudir } \\
\text { 3. No es bueno pero es el único al que puede acudir } \\
\text { 4. No asiste. }\end{array}$ \\
\hline & $\begin{array}{l}\text { Afiliados a algún sistema } \\
\text { de salud }\end{array}$ & $\begin{array}{l}\text { Relación entre el número de personas afiliadas y las que } \\
\text { no. }\end{array}$ \\
\hline & Tiempo de espera & $\begin{array}{l}\text { Cantidad de minutos que tardan en ser atendidos una vez } \\
\text { que llegan al servicio de salud }\end{array}$ \\
\hline
\end{tabular}

Tabla 7. Variables incluidas dentro del indicador de capital humano por dimensiones.

\footnotetext{
${ }^{4}$ Para mayor profundidad en dicha relación entre el tipo de institución al que acuden a atenderse los miembros de un hogar y su condición de pobreza se puede revisar los trabajos de Barquero \& Trejos, 2004; Duhau, 2001; Esposito \& Chiappero-Martinetti, 2010, entre otros.

${ }^{5}$ IMSS. Instituto Mexicano del Seguro Social. ISSSTE. Instituto de Seguridad y Servicios Sociales de los Trabajadores del Estado
} 
El indicador de capital humano demostró tener una buena confianza interna al obtener un alfa de Cronbach de 0.851 , mismo que explica el $30.97 \%$ de la varianza observada en cada una de las variables que lo conforman (Tabla 8). Respecto a sus correlaciones se observó que solamente en algunas variables existe una significancia del 0.01 y 0.05 , siendo en su mayoría inferiores al 0.3 , hecho que indican que pueden no evaluar características similares del capital humano del hogar, y por ende, de sus dimensiones de educación y salud. Sin embargo, la consistencia de las alfas de Cronbach y de las varianzas permiten establecer la consistencia de este indicador (Oviedo \& Campo Arias, 2005).

\begin{tabular}{|c|c|c|c|c|c|c|c|c|c|c|c|}
\hline \multirow{2}{*}{\multicolumn{2}{|c|}{ Variable }} & \multicolumn{8}{|c|}{ Correlaciones de las Variables transformadas } & \multicolumn{2}{|c|}{$\begin{array}{c}\text { Indicadores de } \\
\text { confiabilidad }\end{array}$} \\
\hline & & 1 & 2 & 3 & 4 & 5 & 6 & 7 & 8 & $\begin{array}{c}\text { Alfa de } \\
\text { Cronbach }\end{array}$ & $\begin{array}{c}\% \text { de la } \\
\text { varianza }\end{array}$ \\
\hline 1 & $\begin{array}{l}\text { Primera opción } \\
\text { para atenderse }\end{array}$ & 1 & & & & & & & & \multirow{8}{*}{.851} & \multirow{8}{*}{30.971} \\
\hline 2 & $\begin{array}{l}\text { Razón por que } \\
\text { acude a ese } \\
\text { servicio de salud }\end{array}$ & $\underset{* *}{.381}$ & 1 & & & & & & & & \\
\hline 3 & $\begin{array}{l}\text { Afiliados a algún } \\
\text { sistema de salud }\end{array}$ & $\begin{array}{l}- \\
.189 \\
\end{array}$ & $\begin{array}{l}- \\
.133 \\
\end{array}$ & 1 & & & & & & & \\
\hline 4 & Tiempo de espera & $\begin{array}{l}- \\
.712 \\
* *\end{array}$ & $\begin{array}{l}- \\
.439 \\
* *\end{array}$ & .153 & 1 & & & & & & \\
\hline 5 & $\begin{array}{l}\text { Escolaridad de los } \\
\text { mayores que } \\
\text { aportan al gasto }\end{array}$ & $\begin{array}{l}- \\
.182\end{array}$ & $\begin{array}{l}- \\
.055\end{array}$ & .039 & .131 & 1 & & & & & \\
\hline 6 & $\begin{array}{l}\text { Máximo grado de } \\
\text { estudios }\end{array}$ & .215 & .008 & $\begin{array}{l}- \\
.151 \\
\end{array}$ & $\begin{array}{l}- \\
.119 \\
\end{array}$ & .229 & 1 & & & & \\
\hline 7 & $\begin{array}{l}\text { Cursos } \\
\text { adicionales }\end{array}$ & .041 & $\begin{array}{l}- \\
.068\end{array}$ & $\begin{array}{l}- \\
.138\end{array}$ & $\begin{array}{l}- \\
.030\end{array}$ & .225 & $\begin{array}{l}.743 \\
* *\end{array}$ & 1 & & & \\
\hline 8 & $\begin{array}{l}\text { Mayores que } \\
\text { tomaron cursos } \\
\text { adicionales }\end{array}$ & $\begin{array}{l}- \\
.074\end{array}$ & .045 & $\begin{array}{l}- \\
.016\end{array}$ & $\begin{array}{l}- \\
.010\end{array}$ & .678 & .070 & .072 & 1 & & \\
\hline \multicolumn{12}{|c|}{$N=96$} \\
\hline \multicolumn{12}{|c|}{ **. La correlación es significativa al nivel 0,01 (bilateral). } \\
\hline
\end{tabular}

Tabla 8. Resumen del modelo del indicador de capital humano de los hogares

Es importante mencionar que, para el caso de todos los indicadores, el contar con un valor elevado da muestra de una mayor calidad del capital económico y humano. Con la construcción de estas variables fue que se pudo realizar un análisis de regresión lineal para identificar el efecto que cada uno de los componentes del capital social tienen en el capital económico y humano de los hogares entrevistados. 


\section{El efecto del capital social en el capital económico y humano de los hogares}

Para cumplir con el objetivo de visualizar el efecto del capital social sobre cada uno de los capitales del hogar se realizaron dos análisis diferentes. El primer análisis está relacionado con la variable de capital económico que incluye a su vez ingresos, aspectos laborales y vivienda. El segundo análisis está relacionado con el capital humano e incluye las dimensiones de educación y salud.

Al realizar estos análisis se observó la forma en que los diferentes componentes del capital social -cercanía de relaciones sociales, diversidad de relaciones sociales y grado de cierre de la red social- incidían en las variables de capital económico y humano. Esta estrategia permitió reconocer el efecto que el capital social tiene en el bienestar de los hogares.

\section{Capital social de los hogares y su impacto en el capital económico}

La primera regresión que se realizó fue aquella vinculada con el impacto que los elementos del capital social tuvieron en el capital económico de los hogares. Los resultados dieron muestra de la significancia del modelo al $95 \%$ de confianza. Es decir, las variables del capital social sí generan un efecto significativo en el indicador de capital económico de los hogares. Además, se encontró que estas variables explican el $4.1 \%$ de la varianza del capital económico de los hogares (Tabla 9).

En relación al impacto que las variables del capital social tienen en el capital económico de los hogares, se encontró que la única que resultó significativa, para un intervalo de confianza del $90 \%$, fue la variable de cercanía de relaciones sociales (Tabla 9). A diferencia de lo esperado, en donde las relaciones más lejanas incidirían de forma positiva en la mejora de los aspectos relacionados con el capital económico (Durston, 2002; Narayan, 1999; Woolcock, 1998), el signo negativo en el coeficiente indica que a medida que los hogares tengan en promedio relaciones más cercanas principalmente familiares y amigos con una alta frecuencia temporal y en espacios de interacción cercanos al hogar- que brinden acceso a algún tipo de capital económico, como pueden ser préstamos monetarios, mejoras de la vivienda o incidan en la consecución de algún trabajo el hogar tendrá un mayor nivel en este indicador. 


\begin{tabular}{|c|c|c|c|c|c|c|c|}
\hline \multicolumn{2}{|r|}{ Modelo } & $\begin{array}{l}\text { Suma de } \\
\text { cuadrados }\end{array}$ & $\begin{array}{c}\text { Media } \\
\text { cuadrática }\end{array}$ & $\mathrm{F}$ & \multicolumn{2}{|c|}{ Sig. ${ }^{b}$} & R cuadrado \\
\hline \multirow[t]{3}{*}{1} & Regresión & 7.879 & \multirow{3}{*}{$\begin{array}{r}2.626 \\
.794\end{array}$} & 3.31 & \multirow{3}{*}{\multicolumn{2}{|c|}{$.021 * *$}} & \multirow{3}{*}{.041} \\
\hline & Residual & 182.518 & & & & & \\
\hline & Total & 190.398 & & & & & \\
\hline \multicolumn{8}{|c|}{ Coeficientes $^{a}$} \\
\hline \multirow{2}{*}{\multicolumn{2}{|c|}{ Modelo }} & \multicolumn{2}{|c|}{$\begin{array}{l}\text { Coeficientes no } \\
\text { estandarizados }\end{array}$} & \multicolumn{2}{|c|}{$\begin{array}{l}\text { Coeficientes } \\
\text { tipificados }\end{array}$} & \multirow[t]{2}{*}{$\mathrm{t}$} & \multirow[t]{2}{*}{ Sig. ${ }^{b}$} \\
\hline & & B & Error típ. & Beta & & & \\
\hline \multirow[t]{4}{*}{1} & (Constante) & .388 & .322 & \multirow{2}{*}{\multicolumn{2}{|c|}{-.162}} & 1.203 & .230 \\
\hline & \multirow{3}{*}{$\begin{array}{l}\text { Cercanía de relaciones } \\
\text { sociales de los hogares } \\
\text { Diversidad de las relaciones } \\
\text { sociales de los hogares } \\
\text { Densidad de la red social de } \\
\text { los hogares }\end{array}$} & -.607 & .323 & & & -1.882 & $0.061^{*}$ \\
\hline & & -.257 & .347 & \multicolumn{2}{|c|}{-.062} & -.742 & .459 \\
\hline & & -.948 & .765 & \multicolumn{2}{|c|}{-.083} & -1.239 & .216 \\
\hline \multicolumn{8}{|c|}{$\mathrm{N}=96$} \\
\hline \multicolumn{8}{|c|}{ a. Variable dependiente: Capital Económico } \\
\hline \multicolumn{8}{|c|}{$\begin{array}{l}\text { b. Variables predictoras: (Constante), Cercanía de las relaciones sociales de los hogares, diversidad de } \\
\text { las relaciones sociales de los hogares, y Densidad de la red social de los hogares }\end{array}$} \\
\hline
\end{tabular}

Tabla 9. Resumen del modelo de regresión lineal para el capital económico como variable dependiente

La razón por la cual se puede presentar está situación es por el hecho de que los hogares tienden a incrementar las condiciones de la vivienda por medio de la movilización de sus relaciones sociales más cercanas, ya sea para la participación en la realización de mejoras como puede ser la construcción de nuevos cuartos o la instalación de un nuevo piso (Flores Rodríguez, 2009; Villarreal González, 2010).

En lo que se refiere a los recursos monetarios, la mayoría de los hogares tienden a recurrir a préstamos económicos con familiares y amigos incrementando su capacidad de financiamiento y mejorando el indicador de capital económico (LópezRodríguez \& de la Torre García, 2012; Rodríguez-Oreggia, 2012). Además, cuando el mercado de trabajo presenta altas barreras de entradas, los hogares tienden a movilizar un capital social más cercano y recurren a sus círculos de relaciones más cercanos, como son familiares y amigos (Doeringer \& Piore, 1985; Ioannides \& Loury, 2004; Pistaferri, 1999).

Con base en el análisis presentado se puede decir que las relaciones cercanas son las que inciden de forma positiva en el capital económico. Ahora bien, con respecto a la apertura de las redes sociales se encontró que ésta no fue significativa para el capital económico. 


\section{Capital social de los hogares y su impacto en el capital humano}

Al igual que para el caso del capital económico se realizó una regresión lineal en donde se consideró como variable dependiente al capital humano. Resultado de esta regresión fue que el modelo no resultó significativo en su conjunto, ni tampoco lo hizo para cada una de las variables que integran el concepto del capital social desde la visión minimalista (Tabla 10).

\begin{tabular}{|c|c|c|c|c|c|c|}
\hline & Modelo & $\begin{array}{l}\text { Suma de } \\
\text { cuadrados }\end{array}$ & $\begin{array}{c}\text { Media } \\
\text { cuadrática }\end{array}$ & $\mathrm{F}$ & Sig. ${ }^{b}$ & R cuadrado \\
\hline \multirow[t]{3}{*}{1} & Regresión & .580 & .193 & \multirow[t]{3}{*}{.610} & \multirow[t]{3}{*}{.609} & \multirow[t]{3}{*}{.008} \\
\hline & Residual & 72.899 & \multirow[t]{2}{*}{.317} & & & \\
\hline & Total & 73.479 & & & & \\
\hline \multicolumn{7}{|c|}{ Coeficientes $^{a}$} \\
\hline & \multirow[t]{2}{*}{ Modelo } & \multicolumn{2}{|c|}{$\begin{array}{l}\text { Coeficientes no } \\
\text { estandarizados }\end{array}$} & $\begin{array}{l}\text { Coeficien } \\
\text { tes } \\
\text { tipificado }\end{array}$ & \multirow[t]{2}{*}{$\mathrm{t}$} & \multirow[t]{2}{*}{ Sig. ${ }^{b}$} \\
\hline & & $B$ & Error tín & Beta & & \\
\hline \multirow[t]{4}{*}{1} & (Constante) & .120 & .154 & & .779 & .437 \\
\hline & $\begin{array}{lr}\text { Cercanía } & \text { de } \\
\text { relaciones } & \text { sociales } \\
\text { humano } & \end{array}$ & -.084 & .070 & -.087 & -1.198 & .232 \\
\hline & $\begin{array}{ll}\text { Diversidad de las } \\
\text { relaciones } \\
\text { humano }\end{array}$ & -.022 & .102 & -.015 & -.218 & .828 \\
\hline & $\begin{array}{l}\text { Densidad de la red } \\
\text { social de los hogares }\end{array}$ & -.227 & .493 & -.032 & -.460 & .646 \\
\hline \multicolumn{7}{|c|}{$\mathrm{N}=96$} \\
\hline \multicolumn{7}{|c|}{ a. Variable dependiente: Capital Humano } \\
\hline
\end{tabular}

Tabla 10. Resumen del modelo de regresión lineal para el capital humano como variable dependiente

De los resultados expuestos anteriormente se puede decir que a nivel del capital humano las relaciones sociales no incidieron de forma directa en este indicador, sin embargo, está situación puede no mantenerse al momento de hacer un análisis en donde se tomen por separado cada una de las dimensiones que conforman este capital.

Después de haber realizado el análisis del efecto que el capital social genera en los capitales de los hogares se puede decir que el capital económico tiende a incrementarse en la medida que los hogares presentan relaciones sociales más cercanas, aspecto que habla de la tendencia que tienen los miembros del hogar por acudir a familiares, amigos y vecinos para acceder a préstamos económicos y en algunos casos a fuentes de empleo, además de realizar mejoras relacionados con la 
calidad de la vivienda, como puede ser la construcción de un techo o de un cuarto adicional. En relación al capital humano se observa que ninguna de las variables del capital social tiene una incidencia significativa en todo el indicador, sin embargo, cuando se realiza el análisis a nivel de las dimensiones se pueden encontrar resultados diferentes y que ayudarán a comprender de una mejor forma el fenómeno estudiado, aspecto que será retomado en trabajo posteriores.

En la siguiente sección se presentan algunas conclusiones que versan principalmente sobre el objetivo de este trabajo que fue la construcción de una aproximación al estudio del capital social de los hogares desde una perspectiva minimalista a través de la cual se puedan identificar los efectos que éste genera en diferentes aspectos relacionados con la calidad de vida y bienestar de los mismos.

\section{Conclusiones}

El abordaje que se le dio al capital social en este trabajo permite integrar dos de los elementos que se encuentran presentes en la mayor parte de las definiciones existentes de la aproximación minimalista de este concepto: relaciones sociales caracterizadas por la proximidad de éstas con el actor o ego y la diversidad de las mismas- y red social -definida en función de su grado de apertura o cierre-. Este abordaje permitió observar la forma en que cada una de las variables del capital social incide en el capital económico y humano contribuyendo a la comprensión de la forma en que este capital incide en la mejora de la calidad de vida de los hogares. Este mismo abordaje puede ser utilizado para realizar comparaciones con otras variables de interés relacionadas con hogares que presentan condiciones de vulnerabilidad, rezago social o pobreza.

El estudio del capital social propuesto en este trabajo presenta varios elementos que pueden ser considerados como ventajas en relación a otras aproximaciones similares al momento de estudiar este concepto.

El primer elemento a destacar se encuentra a nivel del entendimiento y tratamiento que se hace del capital social. En esta aproximación no se parte del supuesto de que un mayor número de relaciones sociales de algún tipo o una mayor confianza al interior de una red social es un equivalente a mayores niveles de capital social tal como hacen algunos de los indicadores que existen actualmente en relación a este concepto (Narayan \& Cassidy, 2001; Putnam, 2001; Social Capital Initiative, 1998), 
sino que busca conocer cuáles son los tipos de relaciones y estructuras de la red social de los hogares que ayudan a movilizar determinados recursos.

El partir desde esta perspectiva permite realizar un análisis más fino sobre la forma en que los diferentes componentes de capital social del hogar impactan de forma positiva o negativa en diferentes componentes o variables vinculadas con el hogar, en este caso el capital económico y humano aunque se pueden tomar otros indicadores que sean definidos como claves en otras investigaciones. Los resultados encontrados permiten visualizar por qué el capital social no puede ser medido en términos de indicadores que manifiesten si hay mucho o poco de forma aislada, pues es necesario conocer además el tipo de recurso que moviliza o impacta para saber cuál de todas sus formas mejora las condiciones de vida de los hogares.

Siguiendo esta línea, la utilización de redes personales como forma de recolección de información sobre los componentes del capital social permitió identificar la forma en que los miembros del hogar acceden a otras formas de capitales, en este caso económico y humano, dejando de lado la idea de que contar con un mayor número de relaciones o pertenecer a una red social más cerrada o abierta genera como consecuencia natural una mejora en las condiciones de vida del hogar. Esta estrategia de recolección de información se conjuntó con una estrategia de análisis estadística para generar indicadores de capital social que por su característica continúa favorecen a la realización de análisis estadísticos generales y particulares en donde se delimitan los efectos que este capital tiene de una forma general y particular.

Un segundo elemento a destacar se encuentra a nivel de la construcción de las variables que conforman el capital social del hogar. Estas variables tomaron en consideración no solamente la presencia de determinados tipos de relacione sociales en función de la cercanía cognitiva, tipo de relación o rol social que tienen -tal como se ha hecho al categorizarlas en cierre, puente o enlace-, sino que también incluyó elementos de diversidad de este recurso social, permitiendo visualizar la relevancia que estos aspectos tienen en el acceso a cualquier tipo de capital o recurso.

Asimismo, y como consecuencia de la generación de indicadores, se pudo generar una medición del capital social de corte numérico y no solamente de tipo categórico, hecho que puede facilitar su interpretación y uso en modelos de análisis de tipo cuantitativo. Desde esta perspectiva, el abordaje se encuentra en sintonía con una visión accionalista y minimalista del capital social, en donde se busca comprender las 
estrategias que siguen los actores sociales -en este caso hogares- para satisfacer sus necesidades por medio de la movilización de los activos con los que cuenta.

Es así como esta aproximación, al encontrarse en línea con la perspectiva sociológica accionalista, contribuye al entendimiento de la forma en que los hogares hacen uso de sus activos/recursos sociales para mejorar sus condiciones de vida y satisfacer sus necesidades, contribuyendo a la comprensión sobre la forma en que el capital social se integra como una estrategia de vida que puede contribuir a superar condiciones de pobreza, vulnerabilidad y exclusión social en que se encuentran algunas poblaciones, y que fue uno de los principales alicientes por los cuales el concepto se popularizó en la década de los noventa posicionándolo como un elemento clave en las agendas de desarrollo actuales (Bebbington et al., 2002; Portes \& Landolt, 2000).

A pesar de que uno de los aspectos más relevantes del abordaje teóricometodológico es la visualización de la relevancia que tiene el contar con una determinada distribución del capital social a nivel de la cercanía y diversidad de las relaciones sociales y del grado de apertura de la red social de un determinado hogar en términos de la mejora en sus condiciones de vida; quizá el aspecto más relevante es el identificar cómo esta distribución se presenta en función de los recursos que los miembros del hogar desean movilizar. Es decir, permite establecer que no basta con que los hogares cuenten con un determinado capital social para mejorar sus condiciones de vida, sino que es necesario que lo movilicen de la forma adecuada en función del tipo de capital al que desean acceder para lograr este objetivo.

Por ejemplo, al realizar el análisis en función del capital económico se encontró que las relaciones más cercanas coadyuvan a mejorar este indicador; mientras que para el caso del capital humano, el capital social no genera un cambio significativo en este indicador, hecho que debe de ser validado en un análisis donde se segmenten las dimensiones que lo conforman. La explicación del porqué el capital económico se ve incidido por el capital social fue consecuencia del método de recolección de información utilizado, en donde no solamente se identificaban los diferentes alteri o integrantes de la red social, sino también el contexto bajo el cual surgía este tipo de relación. Esta técnica de recolección de información permitió una interpretación más profunda de los resultados aportados en el análisis estadístico de la información.

Es así como la aproximación expuesta pretende favorecer a la generación de referentes empíricos que contribuyan a la generación de reflexiones y discusiones 
que den como resultados supuestos teóricos sobre la forma en que determinadas distribuciones de capital social inciden en la mejora de las condiciones de vida de hogares y actores sociales de una determinada comunidad o región.

\section{Referencias bibliográficas}

Bartkus, V. O., \& Davis, J. H. (2010). Introduction. In V. O. Bartkus \& J. H. Davis (Eds.), Social capital. Reaching Out, Reaching in (1a ed., pp. 1-14). Massachusetts: Edward Elgar Publishing.

Bebbington, A., Guggenheim, S., Olson, E., \& Woolcock, M. (2002). Exploring Social Capital Debates at the World Bank. Journal of Development Studies, 40(5), 3364.

Bourdieu, P. (1980). Le capital social. Actes de la recherche en sciences sociales, $31(1), 2-3$.

Burt, R. S. (2005). Brokerage and Clousure: An Introduction to Social Capital (p. 279). Oxford University Press.

Coleman, J. S. (1990). Foundations of social theory (1st ed., p. 949). Cambridge, Mass.: Belknap Press of Harvard University Press.

Coleman, J. S. (2008). Constructed Organization : First Principles. Science, 7(January 1991), 7-23.

Collier, P. (2002). Social capital and poverty: a microeconomic perspective. In C. Grootaert \& T. van Bastelaer (Eds.), The role of social capital in development: An empirical assessment (2nd ed., pp. 19-41). Cambridge: Cambridge University Press.

CONEVAL. (2012). Información por municipio. Medición de Pobreza Multidimensional. Retrieved February 01, 2012, from www.coneval.gob.mx

Doeringer, P. B., \& Piore, M. J. (1985). Mercados internos de trabajo y análisis laboral (1st ed., p. 300). Madrid: Servicio de Publicaciones. Ministerio de Trabajo y Seguridad Social.

Durston, J. (2000). Qué es el capital social comunitario? CEPAL Serie Políticas sociales, (38). 
Durston, J. (2002). El capital social campesino en la gestión del desarrollo rural. Díadas, equipos y puentes y escaleras (1a ed., p. 130). Santiago, Chile: Comisión Económica para América Latina y el Caribe (CEPAL).

Esposito, L., \& Chiappero-Martinetti, E. (2010). Multidimensional Poverty: Restricted and Unrestricted Hierarchy Among Poverty Dimensions. Journal of Applied Economics, 13(2), 181-204.

Flores Rodríguez, L. (2009). La Vivienda en México y la población en condiciones de pobreza. México, D.F.

Fuhse, J. A. (2009). The Meaning Structure of Social Networks. Sociological Theory, $27(1), 51-73$.

Gadermann, A. M., Guhn, M., \& Zumbo, B. D. (2012). Estimating ordinal reliability for Likert-type and ordinal item response data: A conceptual, empirical, and practical guide. Practical Assessment, Research \& Evaluation, 17(3), 1-13.

Granovetter, M. S. (1973). The Strength of Weak Ties. (T. S. Huang, A. Nijholt, M. Pantic, \& A. Pentland, Eds.)American J ournal of Sociology, 78(6), 1360-1380.

Grootaert, C., Narayan, D., Jones, V. N., \& Woolcock, M. (2003). Measuring Social Capital An Integrated Questionnaire. Washington.

Hallinan, M. T. (2010). Social capital effects on student outcomes. In V. O. Bartkus \& J. H. Davis (Eds.), Social capital. Reaching Out, Reaching in (1a ed., pp. 145159). Massachusetts: Edward Elgar Publishing.

Hanneman, R. A., \& Riddle, M. (2005). Introduction to Social Networks Methods. Riverside, CA: University of California.

Ioannides, Y. M., \& Loury, L. D. (2004). Job Information Networks, Neighborhood Effects, and Inequality. Journal of Economic Literature, 42(4), 1056-1093.

Krackhardt, D. (1987). Cognitive Social Structures. Social Networks, 9, 109-134.

López-Rodríguez, P., \& de la Torre García, R. (2012). El capital social de los pobres y su acceso a los mercados formales. In P. López-Rodríguez \& I. Soloaga (Eds.), Capital social y política pública en México (1st ed., pp. 219-269). México, D.F.: El Colegio de México, Instituto Nacional de las Mujeres.

McCallister, L., \& Fischer, C. S. (1978). A Procedure for Surveying Personal Networks. Sociological Methods \& Research, 7(2), 131-148. 
McCarty, C., \& Govindaramanujam, S. (2005). A Modified Elicitation of Personal Networks Using Dynamic Visualization. Connections, 26(2), 61-69.

Millán, R., \& Gordon, S. (2004). Capital social: una lectura de tres perspectivas clásicas. Revista Mexicana de Sociología, 66(4), 711-747.

Moser, C. (1998). Reassessing urban poverty reduction strategies: The asset vulnerability framework. World Development, 26(1), 1-19.

Nahapiet, J., \& Ghoshal, S. (1998). Social capital, intellectual capital, and the organizational advantage. Academy of Management Review, 23(2), 242-266.

Narayan, D. (1997). Voices of the poor: poverty and social capital in Tanzania (1st ed., p. 97). Washington: World Bank.

Narayan, D. (1999). Bonds and Bridges: Social Capital And Poverty. Washington.

Narayan, D., \& Cassidy, M. F. (2001). A dimensional approach to measuring Social Capital: Development and Validations of a Social Capital Inventory. Current Sociology, 49(2), 59-102.

Ostrom, E., \& Ahn, T. K. (2009). The meaning of social capital and its link to collective action. In G. T. Svendsen \& G. L. H. Sevensden (Eds.), Handbook of Social Capital. The Troika of Sociology, Political Science and Economics (pp. 17-35). Massachusetts: Edward Elgar Publishing.

Oviedo, H. C., \& Campo Arias, A. (2005). Aproximación al uso del coeficiente Alfa de Cronbach. Revista colombiana de psiquiatría, XXXIV(004), 572-580.

Pistaferri, L. (1999). Informal networks in the italian labor market. Giornale degli economisti e annali di economia, 58, 355-376.

Portales, L. (2012). Capital social como forma de enfrentar a la pobreza y promover el proceso de desarrollo en el Área Metropolitana de Monterrey: el caso de la colonia Fernando Amilpa y sus hogares. Tecnológico de Monterrey.

Portes, A., \& Landolt, P. (2000). Social Capital: Promise and Pitfalls of its Role in Development. J ournal of Latin American Studies, 32(2), 529-547.

Putnam, R. D. (1993). The Prosperous Community. Social Capital and Public Life. The American Prospect, 4(13), 1-11. 
Putnam, R. D. (1995). Tuning In, Tuning Out: The Strange Disappearance of Social Capital in America. PS: Political Science \& Politics, 28(4), 664. doi: $10.2307 / 420517$

Putnam, R. D. (2001). Social Capital: Measurement and Consequences. Canadian J ournal of Policy Research, 2(1), 32.

Robison, L. J., Siles, M. E., \& Schmid, A. A. (2003). El capital social y la reducción de la pobreza: hacia un paradigma maduro. In Capital social y reducción de la pobreza en América Latina y el Caribe: en busca de un nuevo paradigma (pp. 51-114). Santiago, Chile: Comisión Económica para América Latina y el Caribe (CEPAL).

Rodríguez, H., \& Kinto, M. (2010). Crecimiento poblacional y calidad de vida en el Área Metropolitana de Monterrey, 1990-2005. In L. Palacios (Ed.), Cuando México enfrenta la Globalización. Permanencias y cambios en el Área Metropolitana de Monterrey2 (1a ed., pp. 47-66). Monterrey: Universidad Autónoma de Nuevo León.

Rodríguez-Oreggia, E. (2012). Uso de redes sociales y salarios: Evidencia de la ENCASU 2006. In P. López-Rodríguez \& I. Soloaga (Eds.), Capital social y política pública en México (1st ed., pp. 167-188). México, D.F.: El Colegio de México, Instituto Nacional de las Mujeres.

Rojas, M. (2010). The "Measurement of Economic Performance and Social Progress" Report and Quality of Life: Moving Forward. Social Indicators Research, 102(1), 169-180.

Sen, A. (1981). Public action and the quality of life in developing countries. Oxford Bulletin of Economics and Statistics, 43(4), 287-319.

Social Capital Initiative. (1998). The initiative on defining, monitoring and measuring social capital. World. Washington.

Stiglitz, J. E., Sen, A., \& Fitoussi, J.-P. (2008). Report by the Commission on the Measurement of Economic Performance and Social Progress. Sustainable Development (p. 292). Paris.

Sunkel, G. (2003). La pobreza en la ciudad: capital social y políiticas públicas. In R. Atria, M. E. Siles, I. Arriagada, L. J. Robison, \& S. Whiteford (Eds.), Capital 
social y reducción de la pobreza en América Latina y el Caribe: en busca de un nuevo paradigma (pp. 303-338). Santiago, Chile.

Svendsen, G. T., \& Haase Sevendsen, G. L. (2009). The troika of sociology, political science and economics. In G. T. Svendsen \& G. L. Haase Svendsen (Eds.), Handbook of Social Capital. The Troika of Sociology, Political Science and Economics2 (1st ed., pp. 1-13). Cheltenham, UK: Edward Elgar Publishing.

Villarreal González, D. R. (2010). Política de regularización del suelo, producción de vivienda y expansión metropolitana: el caso de la Zona Metropolitana de Monterrey. Producción Económica 2009 (Departamen., p. 30). México, D.F.: UAM-Xochimilco.

Wellman, B., \& Wortley, S. (1990). Different Strokes from Different Folks: Community Ties and Social Support. The American Journal of Sociology, 96(3), 558-588.

Woolcock, M. (1998). Social capital and economic development: Toward a theoretical synthesis and policy framework. Theory and Society, 27, 151-208.

Woolcock, M. (2001). The Place of Social Capital in Understanding Social and economics outcomes. Canadian J ournal of Policy Research, 2(1), 65-88. 